Gut and Liver, Vol. 11, No. 4, July 2017, pp. 528-534

\title{
A Novel Model for Predicting Hepatocellular Carcinoma Development in Patients with Chronic Hepatitis B and Normal Alanine Aminotransferase Levels
}

Dong Hyun Sinn ${ }^{1}$, Jeong-Hoon Lee ${ }^{2}$, Kyunga Kim³ ${ }^{3}$ Joong Hyun Ahn ${ }^{3}$, Ji Hyeon Lee ${ }^{1}$, Jung Hee Kim ${ }^{1}$, Dong Hyeon Lee ${ }^{2}$, Jung-Hwan Yoon ${ }^{2}$, Wonseok Kang ${ }^{1}$, Geum-Youn Gwak ${ }^{1}$, Yong-Han Paik ${ }^{1}$, Moon Seok Choi ${ }^{1}$, Joon Hyeok Lee ${ }^{1}$, Kwang Cheol $\mathrm{Koh}^{1}$, and Seung Woon Paik ${ }^{1}$

${ }^{1}$ Department of Medicine, Samsung Medical Center, Sungkyunkwan University School of Medicine, ${ }^{2}$ Department of Internal Medicine and Liver Research Institute, Seoul National University College of Medicine, and ${ }^{3}$ Biostatistics and Clinical Epidemiology Center, Research Institute for Future Medicine, Samsung Medical Center, Seoul, Korea

Background/Aims: Hepatocellular carcinoma (HCC) can develop in chronic hepatitis $\mathrm{B}(\mathrm{CHB})$ patients with normal alanine aminotransferase (ALT) levels. Therefore, methods that can stratify an individual's HCC risk are needed. Methods: A simple HCC risk score was developed from 971 patients with $\mathrm{CHB}$ who had elevated hepatitis B virus DNA levels $(>2,000$ $\mathrm{IU} / \mathrm{mL}$ ) with normal or mildly elevated ALT levels (<80 U/L). The score was validated from an independent cohort of 507 patients. Results: A 4-point risk scale was developed, with HCC risk ranging from $0 \%$ to $17.8 \%$ at 5 years for the lowest and highest risk scores. The $D^{2} A S$ score had high area under the receiver operating curves (AUROCs) for predicting development of HCC at 3/5 years (0.895/0.884). The calculated AUROCs to predict the development of HCC at $3 / 5$ years were $0.889 / 0.876$ in the validation cohort, with 5 -year $\mathrm{HCC}$ incidence rates ranging from $0 \%$ to $13.8 \%$ at 5 years for the lowest and highest risk scores. Conclusions: The $D^{2} A S$ risk score can play a valuable role in risk stratification and may be useful for guiding clinical decisions for enhanced surveillance or treatment to reduce the HCC risk in $\mathrm{CHB}$ patients with normal or mildly elevated ALT levels. (Gut Liver 2017;11:528-534)

Key Words: Carcinoma, hepatocellular; Hepatitis B; Liver function tests

\section{INTRODUCTION}

Chronic hepatitis B virus (HBV) infection is a major cause of chronic hepatitis, cirrhosis, and hepatocellular carcinoma (HCC) worldwide. $^{1,2}$ Yet, individual risk for liver-related complications varies. Viral, host, and environmental factors influence risks for cirrhosis and HCC. ${ }^{3,4}$ Among these risk factors, HBV DNA level is a key risk factor, as higher HBV DNA level is associated with a higher risk for cirrhosis and HCC, ${ }^{2,3}$ and HBV DNA level can be effectively modulated by antiviral therapy (AVT). ${ }^{2}$ Although effectively suppressing $\mathrm{HBV}$ replication using nucleos(t)ide analogues (NUC) cannot completely eliminate the risk for HCC, ${ }^{5}$ decreasing HBV DNA level by suppressing HBV replication reduces the incidence of HCC and mortality. ${ }^{5-9}$ Therefore, AVT is usually considered to treat patients with chronic hepatitis B (CHB) with elevated HBV DNA levels.

However, not all patients with CHB and elevated HBV DNA levels are recommended for AVT. The American Association for the Study of Liver Diseases (AASLD), the European Association for the Study of the Liver (EASL), and the Asian Pacific Association for the Study of the Liver guidelines suggest that treatment should begin in patients with elevated HBV DNA levels and increased alanine aminotransferase (ALT) concentrations, with slight differences in their recommendations when the HBV DNA concentration is 2,000 to $20,000 \mathrm{IU} / \mathrm{mL}$ and when ALT concentration is between one time or twice the upper limit of normal (ULN) to initiate therapy., ${ }^{3,9}$ The reason for not treating a patient with CHB and an elevated HBV DNA level plus a normal or mildly increased ALT level is straightforward. There is a particular phase in chronic HBV infection where the virus is highly replicative but inflammation is very low. ${ }^{3}$ In this phase, there is minimal risk of disease progression, and treatment dur-

\footnotetext{
Correspondence to: Seung Woon Paik

Department of Medicine, Samsung Medical Center, Sungkyunkwan University School of Medicine, 81 Irwon-ro, Gangnam-gu, Seoul 06351, Korea

Tel: +82-2-3410-3409, Fax: +82-2-3410-6983, E-mail: sw.paik@samsung.com

Received on August 12, 2016. Revised on October 9, 2016. Accepted on November 9, 2016. Published online December 16,2016

pISSN 1976-2283 eISSN 2005-1212 https://doi.org/10.5009/gnl16403

Dong Hyun Sinn and Jeong-Hoon Lee contributed equally to this work as first authors.

@ This is an Open Access article distributed under the terms of the Creative Commons Attribution Non-Commercial License (http://creativecommons.org/licenses/by-nc/4.0) which permits unrestricted non-commercial use, distribution, and reproduction in any medium, provided the original work is properly cited.
} 
ing this phase has no clear clinical benefit. ${ }^{10}$ As a sensitive indicator of hepatocellular damage, serum ALT level is usually low in patients without hepatic inflammation, and high in patients with hepatocyte injury. ${ }^{11}$ The presence of significant fibrosis or liver injury is often reflected by an elevated ALT level. ${ }^{3}$ Therefore, guidelines recommend treating patients with $\mathrm{CHB}$ and an elevated HBV DNA level only when ALT is also elevated.,8,9

Ideally, there should be no disease progression in patients with $\mathrm{CHB}$ who are not indicated for AVT. In other words, there should be no disease progression in CHB patients when ALT level are normal. However, a fair proportion of patients with normal ALT levels have significant fibrosis, ${ }^{12,13}$ and develop serious liver-related complications. ${ }^{14}$ In this study, we assessed the risks for HCC among CHB patients with elevated HBV DNA levels, who were followed up without AVT due to having normal or mildly elevated ALT levels. We developed and validated a risk score for predicting HCC risk in this population, and compared performance to risk factor (ALT and age) or risk score (risk estimation for hepatocellular carcinoma in chronic hepatitis $\mathrm{B}$ [REACH-B] score) that are known to be associated with HCC.

\section{MATERIALS AND METHODS}

\section{Study design, setting, and participants}

The patients population used to develop the risk score (development cohort) consisted of patients who received care at Samsung Medical Center, Seoul, Korea. All patients who had serum HBV DNA level measured using the COBAS TaqMan HBV DNA Test between 2006 (when serum HBV DNA testing with the COBAS TaqMan HBV DNA Test first began) and 2011 $(n=21,812)$ were screened for potential inclusion in the study. The time of this initial HBV DNA measurement was considered the baseline. We enrolled 2,114 patients who met all of the following criteria: (1) age $\geq 18$ years with chronic HBV infection, defined by the presence of hepatitis B surface antigen (HBsAg) in serum for $>6$ months or by clinical history; (2) absence of any of the following clinical indicators of cirrhosis: thrombocytopenia $(<150,000$ platelets/ $\mu \mathrm{L})$, cirrhotic configuration of the liver (nodular liver surface or caudate lobe hypertrophy) and/or splenomegaly confirmed on imaging, or the presence of varices (abnormally enlarged veins detected on upper endoscopy or cross-sectional images) $;^{15}$ (3) no history or current use of interferon or NUC treatment; (4) patients with elevated HBV DNA levels (>20,000 IU/mL for hepatitis B e antigen (HBeAg)-positive patients, and >2,000 IU/mL for HBeAg-negative patients); and (5) patients with normal or mildly increased ALT levels (<80 U/L). These 2,114 patients were further evaluated and excluded from the study if any of following criteria were met: (1) coinfection with hepatitis $C$ virus or human immunodeficiency virus $(n=28)$; (2) follow-up duration $<6$ months (including those who started AVT within 6 months from baseline or who were diagnosed with HCC within 6 months from baseline) ( $n=918)$; (3) other malignancies ( $\mathrm{n}=197)$. Finally, 971 patients were analyzed in the study. The cohort used to validate the risk score (validation cohort) consisted of patients who received care at Seoul National University Hospital, Seoul, Korea, using the same inclusion and exclusion criteria and consisted of 507 patients. This study protocol was reviewed and approved by the Institutional Review Board at Samsung Medical Center, and by the Institutional Review Board at Seoul National University Hospital. Because the study was a retrospective analysis of existing administrative and clinical data, the requirement to obtain informed patient consent was waived by the Institutional Review Board at both centers.

\section{Primary outcome and follow-up}

The primary outcome variable was the diagnosis of HCC during follow-up (monitoring period without AVT). Use of AVT (either interferon or NUC) during follow-up was recorded. Patients who started AVT during follow-up were considered censored at the time AVT was initiated. Elevated ALT concentration $(\geq 80 \mathrm{U} /$ L) with a HBV DNA level $>20,000 \mathrm{IU} / \mathrm{mL}$ for $\mathrm{HBeAg}$-positive patients, and elevated ALT concentration ( $\geq 80 \mathrm{U} / \mathrm{L}$ ) with a HBV DNA level $>2,000 \mathrm{IU} / \mathrm{mL}$ for HBeAg-negative patients at a particular point usually led to the initiation of AVT, according to the Korean insurance reimbursement policy. Follow-up assessments were performed on all patients every 3 to 6 months or more frequently as required for at least 6 months. Person-years were censored on the date of diagnosing HCC, date of initiating AVT, or the last date of follow-up on October 31, 2014, whichever came first.

\section{Study variables}

HCC was diagnosed either by a histological evaluation or clinical imaging. ${ }^{16}$ The following data were collected: age; sex; medical history; ultrasonography and upper endoscopy results; serum platelet count; and HBsAg; HBeAg; HBV DNA; ALT; and aspartate aminotransferase (AST) levels. Patients were also assessed with the REACH-B score, as described previously. ${ }^{17}$ Briefly, sex, age, and ALT, HBeAg, and HBV DNA levels were used to calculate the score. Additionally, those $<30$ years were given a REACH-B score of 0 , whereas those $>65$ years were given score of 6.

\section{Statistical analysis}

For HCC risk prediction, we firstly examined possible risk predictors, including age, gender, HBeAg status, serum HBV DNA level, ALT level, AST level, and platelet level. Note that HBV DNA level was log transformed due to its skewed distribution. While HBV DNA level did not show a significant association with HCC risk in linear manner, we explored nonlinear associations by using a smooth log hazard ratio (HR) plot. A quadratic change in HCC risk was observed as HBV DNA level increases (Supplementary Fig. 1), and hence HBV DNA level and its sec- 
ond order term (i.e., $\log \left(\mathrm{HBV}\right.$ DNA) and $\left[\log (\mathrm{HBV} \text { DNA) }]^{2}\right.$ ) were considered in the risk prediction modeling.

In order to develop a risk prediction model, we used a multivariable Cox regression method with a stepwise selection procedure (Supplementary Table 1). Based on the final prediction model, called as $\mathrm{D}^{2} \mathrm{AS}$ model, HCC risk was estimated with the equation: $2.9325 \times \log (\mathrm{HBV}$ DNA IU/mL)$0.10527 \times[\log (\mathrm{HBV} \mathrm{DNA} \mathrm{IU} / \mathrm{mL})]^{2}+-1.27223 \times(2$ if female and 1 if male)+0.07013 $\times$ age (years). HR with its 95\% confidence interval (CI) was estimated for each of the selected risk predictors. Using an independent cohort data, we externally validated the discrimination performance of the newly developed $\mathrm{D}^{2} \mathrm{AS}$ model, and compared it to other risk predictors, such as REACH$\mathrm{B}$, and to age and ALT. The time-dependent area under receiver operating characteristic (AUROC) curve was used to assess discrimination performance.

For easy interpretation, we further formulated $\mathrm{D}^{2} \mathrm{AS}$ risk scores based on the newly developed $\mathrm{D}^{2} \mathrm{AS}$ model considering ranges of age (20 to 90 years) and HBV DNA levels (2,000 to $500,000,000 \mathrm{IU} / \mathrm{mL}): \mathrm{D}^{2} \mathrm{AS}$ risk score $=[(\mathrm{HCC}$ risk estimated in the $\mathrm{D}^{2} \mathrm{AS}$ model) -15$] / 5 \times 2$. Four risk groups were constructed according to the score: very low risk $=\mathrm{D}^{2} \mathrm{AS}$ risk score $<1$; low risk $=\mathrm{D}^{2} \mathrm{AS}$ risk score $\geq 1$ and $<2$; intermediate risk $=\mathrm{D}^{2} \mathrm{AS}$ risk score $\geq 2$ and $<2.5$; high risk $=\mathrm{D}^{2} \mathrm{AS}$ risk score $\geq 2.5$, respectively. The Kaplan-Meier method was used to see the cumulative HCC incidence rate in each group.

For subgroup analysis, patients in the derivation and validation cohort were merged and subgrouped into $\mathrm{HBeAg}(+)$ and HBeAg (-) patients according to the presence of $\mathrm{HBeAg}$, and sub-grouped into normal or mildly elevated ALT levels, according to the known ALT cutoff (34 IU/L for men and $25 \mathrm{IU} / \mathrm{L}$ for women), ${ }^{18}$ which was based on Nationally representative population. Statistical significance was defined as $\mathrm{p}$-value $<0.05$.

\section{RESULTS}

\section{Patient characteristics and incidence of the HCC}

The baseline characteristics of the patients are shown in Table 1. During the median 4.5 year follow-up (range, 1.0 to 8.7 years), 26 patients were newly diagnosed with HCC. The cumulative HCC incidence rate at 5 years was 3.2\%. Patients who developed HCC were older, more often male, and had a slightly higher AST level but a lower platelet count. The proportion of patients with elevated ALT was similar between those who developed HCC and those who did not. The REACH-B score also higher in patients who developed HCC (Table 1). The baseline characteristics of the validation cohort were similar to those of the derivation cohort in terms of age, sex, and ALT and AST levels, however, fewer patients were HBeAg positive, HBV DNA levels were lower, and baseline platelet counts were higher (Table $1)$.

\section{Performance of the $D^{2} A S$ score}

The performance of the $\mathrm{D}^{2} \mathrm{AS}$ score is shown in Table 2. The time-dependent AUROCs of the $\mathrm{D}^{2}$ AS score at 3/5 years $(95 \%$ CI) was 0.895 (0.823 to 0.966$) / 0.884$ (0.812 to 0.955$)$ in the derivation cohort and 0.889 (0.796 to 0.983$) / 0.876$ (0.789 to $0.963)$ in the validation cohort. When new model was compared to other risk factor or risk scores in the validation set (Table 3), AUROCs at each time point were constantly higher in the new model than those for age, ALT, and REACH-B score, although the difference did not reach statistical significance at 3 years for age and at 3 and 6 years for REACH-B. In addition, we compared goodness-of-fit measures with both derivation and validation sets. $\mathrm{D}^{2} \mathrm{AS}$ showed better concordance (0.861 in the derivation set and 0.878 in the validation set) than REACH-B (0.777 in the derivation set and 0.759 in the validation set), age (0.735 in the derivation set and 0.718 in the validation set) and

Table 1. Baseline Characteristics of Cohort

\begin{tabular}{lcccccc}
\hline \multicolumn{1}{c}{ Characteristic } & $\begin{array}{c}\text { Derivation } \\
(\mathrm{n}=971)\end{array}$ & $\begin{array}{c}\text { No HCC } \\
(\mathrm{n}=945)\end{array}$ & $\begin{array}{c}\text { HCC } \\
(\mathrm{n}=26)\end{array}$ & $\begin{array}{c}\text { HCC vs No HCC } \\
\mathrm{p} \text {-value }\end{array}$ & $\begin{array}{c}\text { Validation } \\
(\mathrm{n}=507)\end{array}$ & $\begin{array}{c}\text { Derivation vs validation } \\
\mathrm{p} \text {-value }\end{array}$ \\
\hline Age, yr & $42.6 \pm 10.6$ & $42.4 \pm 10.5$ & $51.4 \pm 10.8$ & $<0.001$ & $42.2 \pm 12.4$ & 0.46 \\
Male sex & $564(58.1)$ & $544(57.6)$ & $20(76.9)$ & 0.048 & $283(55.8)$ & 0.40 \\
Hepatitis B e antigen positive & $547(56.3)$ & $535(56.6)$ & $12(46.2)$ & 0.28 & $202(41.6)^{\dagger}$ & $<0.001$ \\
HBV DNA levels, IU/mL & $5.95 \pm 1.84$ & $5.96 \pm 1.85$ & $5.74 \pm 1.27$ & 0.55 & $5.71 \pm 1.79$ & 0.014 \\
ALT, U/L & $34(23-49)$ & $34(23-49)$ & $37(26-52)$ & 0.28 & $33(22-49)$ & 0.49 \\
$\quad$ Elevated ALT* & $582(59.9)$ & $565(59.8)$ & $17(65.4)$ & 0.68 & $375(74.0)$ & 0.60 \\
AST, U/L & $29(23-37)$ & $29(23-37)$ & $31(26-38)$ & 0.076 & $28(23-37)$ & 0.32 \\
Platelet, $\times 10^{3} / \mu \mathrm{L}$ & $205(182-238)$ & $206(183-239)$ & $186(170-214)$ & 0.023 & $213(185-250)$ & 0.015 \\
REACH-B score & $10(8-11)$ & $10(8-11)$ & $12(10-13)$ & $<0.001$ & $9(7-11)$ & 0.30
\end{tabular}

Data are presented as mean $\pm \mathrm{SD}$, number (\%), or median (quartile range).

HCC, hepatocellular carcinoma; HBV, hepatitis B virus; ALT, alanine aminotransferase; AST, aspartate aminotransferase; REACH-B, risk estimation for hepatocellular carcinoma in chronic hepatitis B.

*Elevated ALT ( $\geq 35 \mathrm{IU} / \mathrm{L}$ for male and $\geq 24 \mathrm{IU} / \mathrm{L}$ for female); ${ }^{\dagger}$ Missing in 21 patients. 
Table 2. Performance of $\mathrm{D}^{2} \mathrm{AS}$ Score and Validation in Independent Cohort

\begin{tabular}{|c|c|c|c|c|c|c|c|c|c|}
\hline \multicolumn{5}{|c|}{ Derivation $(\mathrm{n}=971)$} & \multicolumn{5}{|c|}{ Validation $(\mathrm{n}=507)$} \\
\hline Year & HCC & Survivors & Censored & AUROC (95\% CI) & Year & HCC & Survivors & Censored & AUROC (95\% CI) \\
\hline 2 & 10 & 727 & 234 & $0.878(0.799-0.957)$ & 2 & 7 & 420 & 80 & $0.942(0.901-0.984)$ \\
\hline 3 & 11 & 584 & 376 & $0.895(0.823-0.966)$ & 3 & 11 & 382 & 114 & $0.889(0.796-0.983)$ \\
\hline 4 & 13 & 460 & 498 & $0.884(0.817-0.949)$ & 4 & 13 & 353 & 141 & $0.880(0.798-0.962)$ \\
\hline 5 & 19 & 331 & 621 & $0.884(0.812-0.955)$ & 5 & 13 & 265 & 229 & $0.876(0.789-0.963)$ \\
\hline 6 & 21 & 229 & 721 & $0.882(0.815-0.948)$ & 6 & 15 & 127 & 365 & $0.778(0.663-0.892)$ \\
\hline
\end{tabular}

HCC, hepatocellular carcinoma; AUROC, area under the receiver operating characteristic curve; CI, confidence interval.

Table 3. Comparison of Area under the Receiver Operating Characteristic of $\mathrm{D}^{2} \mathrm{AS}$ Score with Other Risk Factors or Risk Scores in the Validation Set

\begin{tabular}{ccccccccc}
\hline Year & $\mathrm{D}^{2}$ AS score & Age & p-value & ALT & p-value & REACH-B & p-value \\
\hline 2 & 0.942 & 0.807 & 0.014 & 0.684 & 0.003 & 0.869 & 0.006 \\
3 & 0.889 & 0.765 & 0.081 & 0.696 & 0.020 & 0.817 & 0.087 \\
4 & 0.880 & 0.731 & 0.013 & 0.664 & 0.002 & 0.750 & 0.016 \\
5 & 0.876 & 0.729 & 0.021 & 0.665 & 0.003 & 0.733 & 0.010 \\
6 & 0.778 & 0.612 & 0.019 & 0.563 & 0.027 & 0.664 & 0.291 \\
\hline
\end{tabular}

ALT, alanine aminotransferase; REACH-B, risk estimation for hepatocellular carcinoma in chronic hepatitis B.

Table 4. Risk of Hepatocellular Carcinoma According to $D^{2}$ AS Score

\begin{tabular}{|c|c|c|c|c|c|c|c|c|}
\hline \multirow{3}{*}{$\begin{array}{l}\text { Risk group by } \\
D^{2} \mathrm{AS} \text { score }\end{array}$} & \multicolumn{4}{|c|}{ Derivation } & \multicolumn{4}{|c|}{ Validation } \\
\hline & Very low & Low & Intermediate & High & Very low & Low & Intermediate & High \\
\hline & $<1.0$ & $1.0-1.9$ & $2.0-2.4$ & $\geq 2.5$ & $<1.0$ & $1.0-1.9$ & $2.0-2.4$ & $\geq 2.5$ \\
\hline No. & 48 & 537 & 246 & 140 & 38 & 268 & 106 & 92 \\
\hline Risk score & $0.8 \pm 0.1$ & $1.5 \pm 0.2$ & $2.2 \pm 0.1$ & $2.8 \pm 0.2$ & $0.8 \pm 0.1$ & $1.5 \pm 0.2$ & $2.2 \pm 0.1$ & $2.8 \pm 0.2$ \\
\hline HCC & 0 & $3(0.6)$ & $6(2.4)$ & $17(12.1)$ & 0 & $1(0.4)$ & $2(1.8)$ & $12(13.0)$ \\
\hline \multicolumn{9}{|c|}{ Cumulative incidence rate, $\%$} \\
\hline $2 \mathrm{yr}$ & 0 & 0.2 & 0.9 & 6.2 & 0 & 0 & 0 & 8.4 \\
\hline $3 \mathrm{yr}$ & 0 & 0.2 & 0.9 & 7.3 & 0 & 0.5 & 0 & 12.5 \\
\hline $4 \mathrm{yr}$ & 0 & 0.2 & 1.6 & 9.0 & 0 & 0.5 & 1.1 & 13.8 \\
\hline $5 \mathrm{yr}$ & 0 & 0.7 & 2.7 & 17.8 & 0 & 0.5 & 1.1 & 13.8 \\
\hline $6 \mathrm{yr}$ & 0 & 0.7 & 4.3 & 21.7 & 0 & 0.5 & 3.4 & 15.7 \\
\hline
\end{tabular}

Data are presented as mean \pm SD or number $(\%)$.

HCC, hepatocellular carcinoma.

ALT ( 0.627 in the derivation set and 0.670 in the validation set).

\section{Cumulative incidence of HCC according to $D^{2} A S$ score}

The cumulative incidence of HCC according to $\mathrm{D}^{2} \mathrm{AS}$ risk score are shown in Table 4 . The cumulative incidence rates of HCC at 3/5 years were $0 \% / 0 \%, 0.2 \% / 0.7 \%, 0.9 \% / 2.7 \%$, and $7.3 \% / 17.8 \%$ for the very low, low, intermediate, and high $\mathrm{D}^{2} \mathrm{AS}$ scores, respectively (Fig. 1A). In the validation cohort, the cumulative incidence rates of HCC at 3/5 years were $0 \% / 0 \%$, $0.5 \% / 0.5 \%, 0 \% / 1.1 \%$, and $12.5 \% / 13.8 \%$ for very low, low, intermediate, and high $\mathrm{D}^{2} \mathrm{AS}$ scores, respectively (Fig. 1B).

\section{Performance $D^{2} A S$ score in subgroup}

The 5-year cumulative HCC incidence rate was 2.0\% and 3.8\% for those with $\mathrm{HBeAg}(+)$ and $\mathrm{HBeAg}(-)$ patients $(\mathrm{p}=0.12)$. The time-dependent AUROCs of the $\mathrm{D}^{2} \mathrm{AS}$ score at 3/5 years $(95 \%$ CI) was $0.908(0.827-0.989) / 0.878(0.758-0.998)$ in the HBeAg $(+)$ patients and $0.871(0.772-0.970) / 0.858(0.788-0.929)$ in the HBeAg (-) patients. The 5-year cumulative HCC incidence rate was $1.2 \%$ and $4.6 \%$ for those with normal and mildly elevated ALT levels $(\mathrm{p}=0.036)$. The time-dependent AUROCs of the $\mathrm{D}^{2} \mathrm{AS}$ score at 3/5 years $(95 \% \mathrm{CI})$ was $0.864(0.749-0.978) / 0.894$ 
A

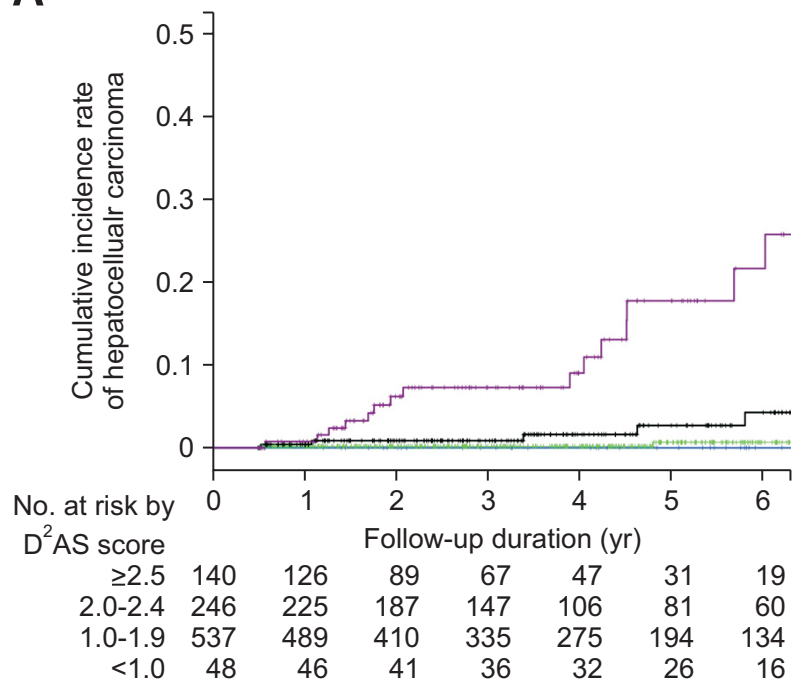

B

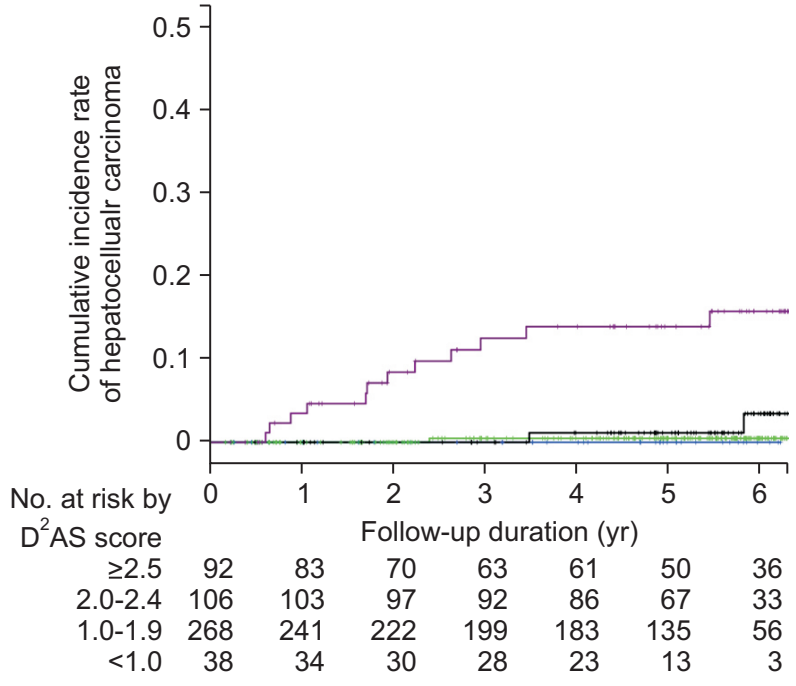

Fig. 1. Cumulative incidence of hepatocellular carcinoma according to the $D^{2} A S$ score. The hepatocellular carcinoma risk was high for those with a high $\mathrm{D}^{2} \mathrm{AS}$ score $\left(\geq 2.5\right.$ ). Purple, black, green and blue represent those with $\mathrm{D}^{2} \mathrm{AS}$ scores of $\geq 2.5,2.0$ to $2.4,1.0$ to 1.9 , and $<1.0$, respectively. (A) Derivation cohort and (B) validation cohort.

(0.799-0.988) in patients with normal ALT and 0.886 (0.8070.965)/0.838 (0.758-0.919) in patients with mildly elevated ALT levels. The AUROCS of $\mathrm{D}^{2} \mathrm{AS}$ scores at each time point was higher than age, ALT, and REACH-B in all subgroups analyzed (HBeAg (+), HBeAg (-), normal ALT and mildly elevated ALT levels), although the difference was not statistically significant in some time point (Supplementary Table 2).

\section{DISCUSSION}

In this study, the 5-year cumulative HCC incidence rate was $3.2 \%$ in the derivation cohort and 3.0\% in validation cohort, indicating that a minor but significant proportion of patients with CHB and elevated HBV DNA levels develop HCC, although they have normal or mildly elevated ALT levels. Consistent to this study, Chao et al. ${ }^{19}$ showed that approximately one-fifth of patients with $\mathrm{CHB}$ and ALT $\leq 40 \mathrm{IU} / \mathrm{L}$ have hepatic fibrosis in their meta-analysis. HCC has been reported in patients with $\mathrm{CHB}$ and normal ALT levels. ${ }^{20}$ These findings indicate that patients with CHB and elevated HBV DNA levels are at risk for developing HCC even when their ALT levels are normal or mildly elevated. However, given the low incidence rate of HCC in this population $(<1.0 \%$ per year), methods that can further stratify individuals risk for HCC are needed.

In this aspect, the newly developed $\mathrm{D}^{2} \mathrm{AS}$ score can play a valuable role for $\mathrm{CHB}$ patients with normal or mildly elevated ALT levels. In CHB patients, development of HCC is multifactorial process with complex interaction between virus, host and environmental exposures. ${ }^{1}$ Among viral factors, increase in HBV DNA level has been consistently reported to be associated with increased HCC risk. ${ }^{17,21}$ However, during the natural course of chronic HBV infection, it is also well-known that when patients are in its immune tolerant phase, HCC risk is low despite of very high HBV DNA levels. ${ }^{21}$ In this study, we could also notice an increase in HCC risk with increase in HBV DNA levels to a certain point, than decrease in HCC risk with a further increase in HBV DNA levels (Supplementary Fig. 1). In order to statistically reflect this quadratic phenomenon, we have added the second order term of HBV DNA levels, which is a way to model presumable nonlinear relationship between the variable and log hazard. ${ }^{22}$ ALT levels are known to be associated with HCC risk, ${ }^{3}$ and the 5-year cumulative HCC incidence rate was higher for those with mildly elevated ALT levels than normal ALT levels in this study as well (4.6\% vs $1.2 \%, \mathrm{p}=0.036)$. We explored whether ALT is a significant factor for HCC development in our cohort using ALT as a continuous variable as well as a categorized variable (Supplementary Table 1). In final model selection, the ALT was excluded in stepwise selection. In addition, we fitted another model that included ALT into the final model, and found that ALT was not significant $(p=0.5499)$ while the first and second order terms of HBV DNA levels were still significant (data not shown). We only included those with normal or mildly elevated ALT levels, which may explain why ALT level was not selected in the final model. Finally, a simple risk prediction model using age, sex and HBV DNA levels was developed which could stratify HCC risk in CHB patients with normal or mildly elevated ALT levels. The risk score was validated from an independent cohort at another hospital, which showed same findings. The $\mathrm{D}^{2} \mathrm{AS}$ score was simplified into a four-category risk scale for easy interpretation. No cases of HCC were observed up to 5-year of follow-up in patients with a very low $\mathrm{D}^{2} \mathrm{AS}$ score $(<1)$, and HCC was rare $(<1 \%$ up to 5 -year) in patients with a 
low $\mathrm{D}^{2} \mathrm{AS}$ score (1 to 2). The HCC risk was intermediate when patients has high $\mathrm{D}^{2} \mathrm{AS}$ score (2 to 2.5), and was high when the risk score was $\geq 2.5$. Therefore, patients with a $\mathrm{D}^{2} \mathrm{AS}$ score $\geq 2.5$ require careful monitoring for HCC development, and may require prompt AVT to reduce HCC risk.

There are some other ways to risk-stratify individuals risk for disease progression in CHB patients with normal ALT levels. The AASLD guideline recommend to consider age, family history of HCC, previous treatment history and presence of extrahepatic manifestations for CHB patients with normal ALT levels. ${ }^{3}$ The EASL guideline recommends lower ULN for ALT to define healthy ALT levels. ${ }^{8}$ In this study, we could notice higher AUROC for the new model than age or ALT. REACH-B can also be used to risk stratify individuals risk for HCC in CHB patients. ${ }^{21}$ When compared, the newly developed $\mathrm{D}^{2} \mathrm{AS}$ score showed better AUROCs than REACH-B. Also, the goodness-of-fit measures were better in the new model.

In this study, we included individuals with ALT less than 80 IU/L, regardless of sex. Our inclusion criteria reflected regional guideline, ${ }^{21}$ where close monitoring is recommended for patients who has ALT levels less than two times of ULN. This inclusion criteria was to select patients in whom CHB guideline recommend close monitoring over treatment. However, some studies have shown that the healthy ALT threshold should be lower than the currently accepted threshold, and that sex-specific thresholds should be applied. ${ }^{23-25}$ The healthy ALT thresholds should also consider ethnicity, so we used the ALT cutoff of Korean (34 IU/L for men and $25 \mathrm{IU} / \mathrm{L}$ for women), which involved a nationally representative healthy cohort from the Fourth Korea National Health and Nutrition Examination Survey. ${ }^{18}$ We found that the HCC incidence rate was lower for patients with a normal ALT level than those with a mildly elevated ALT level. However, HCC development was still observed for patients with normal ALT levels. In subgroup analysis, stratified by HBeAg or ALT levels, the $\mathrm{D}^{2} \mathrm{AS}$ score showed high AUROCs for HBeAg(+) patients, HBeAg (-) patients, those with normal ALT levels, and those with mildly elevated ALT levels (Supplementary Table 2).

This study has some limitations that should be mentioned. The overall follow-up duration for the analyzed patients was 4.5 years, which may be relative short to definitely reflect HCC incidence in this population. Almost all Korean patients with $\mathrm{CHB}$ are infected with HBV genotype $\mathrm{C},{ }^{26}$ which progresses more rapidly to HCC than that of the other genotypes. Therefore, the relevance of our findings to other HBV genotypes remains to be determined. Histological data and noninvasive study data reflecting fibrosis (e.g., Fibroscan) were missing. As fibrosis is an important risk factor for the development of HCC, ${ }^{27}$ our data may need to be compared to a score that incorporates fibrotic burden. For this study, those with evidence of cirrhosis was excluded. However, noninvasive prediction of cirrhosis has shortcomings that some patients with cirrhosis might have been included. Not all risk factors for HCC, such as family history of
HCC, smoking, alcohol consumption, viral mutation, etc., were tested.

Nevertheless, our data are informative for clinicians in everyday practice. It is clear that a small but significant proportion of patients with CHB and elevated HBV DNA levels develop HCC, although they have normal or mildly increased ALT level. Several measures can be used to further risk-stratify risk for disease progression. ALT level, age, and REACH-B score can be considered as an additional factor to guide clinicians regarding regular surveillance, enhanced surveillance, or prompt AVT. The newly developed $\mathrm{D}^{2} \mathrm{AS}$ score is composed of only three factors (HBV DNA, age, and sex) making is easy to calculate, and can be widely used in clinical practice, and showed superior performance than other risk scores in the prediction of HCC in $\mathrm{CHB}$ patients with elevated HBV DNA levels plus normal or mildly increased ALT levels. The $\mathrm{D}^{2} \mathrm{AS}$ risk score can play a valuable role in risk stratification, and may be useful to guide clinical decisions for enhanced surveillance or treatment to reduce HCC risk in this population.

\section{CONFLICTS OF INTEREST}

No potential conflict of interest relevant to this article was reported.

\section{ACKNOWLEDGEMENTS}

Guarantor of the article: S.W.P.; study design and supervision: D.H.S., S.W.P., J.H.L.; statistical analysis: D.H.S., K.K., J.H.A.; writing of the draft manuscript: D.H.S., J.H.L.; data collection: J.H.L., J.H.K., D.H.L., J.H.Y., W.K., G.Y.G., Y.H.P., M.S.C., J.H.L., K.C.K.; critical revision of the manuscript: D.H.L., J.H.Y., W.K., G.Y.G., Y.H.P., M.S.C., J.H.L., K.C.K., S.W.P. All authors approved the final version of the manuscript.

\section{REFERENCES}

1. Trépo C, Chan HL, Lok A. Hepatitis B virus infection. Lancet 2014;384:2053-2063.

2. Korean Association for the Study of the Liver. KASL clinical practice guidelines: management of chronic hepatitis B. Clin Mol Hepatol 2012;18:109-162.

3. Terrault NA, Bzowej NH, Chang KM, et al. AASLD guidelines for treatment of chronic hepatitis B. Hepatology 2016;63:261-283.

4. Yim HJ, Lok AS. Natural history of chronic hepatitis B virus infection: what we knew in 1981 and what we know in 2005. Hepatology 2006;43(2 Suppl 1):S173-S181.

5. Cho JY, Paik YH, Sohn W, et al. Patients with chronic hepatitis B treated with oral antiviral therapy retain a higher risk for HCC compared with patients with inactive stage disease. Gut 2014;63:1943-1950.

6. Liaw YF, Sung JJ, Chow WC, et al. Lamivudine for patients with 
chronic hepatitis B and advanced liver disease. N Engl J Med 2004;351:1521-1531.

7. Lim YS, Han S, Heo NY, Shim JH, Lee HC, Suh DJ. Mortality, liver transplantation, and hepatocellular carcinoma among patients with chronic hepatitis B treated with entecavir vs lamivudine. Gastroenterology 2014;147:152-161.

8. European Association for the Study of the Liver. EASL clinical practice guidelines: management of chronic hepatitis B virus infection. J Hepatol 2012;57:167-185.

9. Liaw YF, Kao JH, Piratvisuth T, et al. Asian-Pacific consensus statement on the management of chronic hepatitis B: a 2012 update. Hepatol Int 2012;6:531-561.

10. Chan HL, Chan CK, Hui AJ, et al. Effects of tenofovir disoproxil fumarate in hepatitis B e antigen-positive patients with normal levels of alanine aminotransferase and high levels of hepatitis B virus DNA. Gastroenterology 2014;146:1240-1248.

11. Pratt DS, Kaplan MM. Evaluation of abnormal liver-enzyme results in asymptomatic patients. N Engl J Med 2000;342:12661271.

12. Kumar M, Sarin SK, Hissar S, et al. Virologic and histologic features of chronic hepatitis B virus-infected asymptomatic patients with persistently normal ALT. Gastroenterology 2008;134:13761384.

13. Lai M, Hyatt BJ, Nasser I, Curry M, Afdhal NH. The clinical significance of persistently normal ALT in chronic hepatitis B infection. J Hepatol 2007;47:760-767.

14. Tong MJ, Hsien C, Hsu L, Sun HE, Blatt LM. Treatment recommendations for chronic hepatitis B: an evaluation of current guidelines based on a natural history study in the United States. Hepatology 2008;48:1070-1078.

15. Yang JD, Kim WR, Coelho R, et al. Cirrhosis is present in most patients with hepatitis B and hepatocellular carcinoma. Clin Gastroenterol Hepatol 2011;9:64-70.

16. Lok AS, McMahon BJ. Chronic hepatitis B: update 2009. Hepatology 2009;50:661-662.

17. Yang HI, Yuen MF, Chan HL, et al. Risk estimation for hepatocel- lular carcinoma in chronic hepatitis B (REACH-B): development and validation of a predictive score. Lancet Oncol 2011;12:568574.

18. Park HN, Sinn DH, Gwak GY, et al. Upper normal threshold of serum alanine aminotransferase in identifying individuals at risk for chronic liver disease. Liver Int 2012;32:937-944.

19. Chao DT, Lim JK, Ayoub WS, Nguyen LH, Nguyen MH. Systematic review with meta-analysis: the proportion of chronic hepatitis B patients with normal alanine transaminase $\leq 40 \mathrm{IU} / \mathrm{L}$ and significant hepatic fibrosis. Aliment Pharmacol Ther 2014;39:349358.

20. Kumada T, Toyoda H, Kiriyama S, et al. Incidence of hepatocellular carcinoma in patients with chronic hepatitis B virus infection who have normal alanine aminotransferase values. J Med Virol 2010;82:539-545.

21. Korean Association for the Study of the Liver. KASL clinical practice guidelines: management of chronic hepatitis B. Clin Mol Hepatol 2016;22:18-75.

22. Clark TG, Bradburn MJ, Love SB, Altman DG. Survival analysis part IV: further concepts and methods in survival analysis. Br J Cancer 2003;89:781-786.

23. Prati D, Taioli E, Zanella A, et al. Updated definitions of healthy ranges for serum alanine aminotransferase levels. Ann Intern Med 2002;137:1-10

24. Kang HS, Um SH, Seo YS, et al. Healthy range for serum ALT and the clinical significance of "unhealthy" normal ALT levels in the Korean population. J Gastroenterol Hepatol 2011;26:292-299.

25. Lee JK, Shim JH, Lee HC, et al. Estimation of the healthy upper limits for serum alanine aminotransferase in Asian populations with normal liver histology. Hepatology 2010;51:1577-1583.

26. Bae SH, Yoon SK, Jang JW, et al. Hepatitis B virus genotype C prevails among chronic carriers of the virus in Korea. J Korean Med Sci 2005;20:816-820.

27. Jung KS, Kim SU, Song K, et al. Validation of hepatitis B virusrelated hepatocellular carcinoma prediction models in the era of antiviral therapy. Hepatology 2015;62:1757-1766. 